\title{
Phagocytosis of Hemozoin (Native and Synthetic Malaria Pigment), andPlasmodium falciparum Intraerythrocyte-Stage Parasites by Human and Mouse Phagocytes
}

\section{Piero Olliaro, MD}

UNDP/WorldBank/WHO Special Programme for Research and Training in Tropical Diseases (TDR),

Geneva, Switzerland

\section{Luciano Lombardi, PhD and Simona Frigerio, PT}

Dipartimento di Oncologia Sperimentale, Instituto Nazionale Tumori, Milano, Italy

\section{Nicoletta Basilico, PhD and Donatella Taramelli, PhD}

Istituto di Microbiologia, CSSON, CNR, Universitá di Milano, Milano, Italy

\section{Diego Monti, PhD}

Dipartimento de Chimica Organica e Industriale, CSSON, CNR, Universitá di Milano, Milano, Italy
Hemozoin, the detoxification product of hemoglobin heme, piles up as electron-dense material in the food vacuole (FV) of intraerythrocytic malaria parasites (malaria pigment). In infected individuals, pigment is internalized by both circulating and resident phagocytes, thus modulating their functions. Synthetic $\beta$-hematin, prepared in vitro from hematin (ferriprotoporphyrin IX hydroxide) in acidic condition, is spectroscopically identical to hemozoin. In this electron microscopy study, native and synthetic hemozoin also prove to be morphologically indistinguishable (large polygonal crystals with apparent transverse banding) and to undergo the same process when internalized by phagocytes (primarily a direct uptake of crystals, similar to what is described for asbestos fibers). On the contrary, whole parasites appear to follow a classical endocytic pathway. This suggests that there may be differences between the ingestion of free particles and whole parasites in terms of modulation of phagocytes' functions.

Keywords $\beta$-hematin, hemozoin, malaria, malaria pigment, phagocytosis, red blood cells
Hemozoin (malaria pigment) is an insoluble material resulting from the polymerization of heme (Ferri-protoporphyrin (X), the prosthetic part of hemoglobin. It resides in the food vacuole (FV) of the malaria parasite, a lysosome-like organelle. The malaria pigment contains, in addition to the hemozoin polymer, variable amounts of lipids and proteins [1-5]. Hemozoin can also be synthesized in vitro from hematin in acidic conditions; the synthetic polymer, $\beta$-hematin, retains the chemical characteristics of native hemozoin $[3,6,7]$.

Hemozoin is also found in various cells of the infected host, both in the tissues where parasites sequester and in the peripheral circulation. It accumulates primarily in macrophages, monocytes, and neutrophils, as a result of the phagocytosis of either

Received 14 October 1999; accepted 25 November 1999.

We are thankful to Prof. M. Ghione and Prof. Omodeo Sale for invaluable advice and helpful comments; to Prof. S. Picot, Université de Lyon, France, for providing purified hemozoin; Mr. Mario Azzini for the microphotographs.

This work was supported in part by UNDP/World Bank/WHO Special Programme for Research and Training on Tropical Diseases, Geneva, (CH) N. 97-0115/98-0363 and by the Ministero Italiano dell'Universitá e della Ricerca Scientifica e Technologica, Progetti di Rilevante Interesse Nazionale,

Cofinanziamento 1997, 1999.

Address Correspondence to Prof. Donatella Taramelli, Istituto di Microbiologia, Via Pascal 36, 20133 Milano, Italy.

E-mail: Donatella.Taramelli@unimi.it intraerythrocytic malaria parasites, "residual bodies" (the remnant of a parasite after the schizogonic cycle is completed and merozoites are released), or, possibly, free heme [8-14]. The black pigment appears to persist in the cells, possibly for their entire life-span. The amount of pigment in the mononuclear phagocyte system appears to be correlated with the duration of infection [11-13].

Hemozoin uptake occurs in vitro too. Various cell types prove able to internalize hemozoin in vitro, including monocytes [15, 16], macrophages, and endothelial cells $[5,17]$; however, to our knowledge, the process has not been adequately documented microscopically thus far.

This electron microscopy study was set up to compare the morphology of natural and synthetic pigment, and to document the uptake and intracellular distribution of pigment resulting from the ingestion of pigment crystals or whole parasites.

\section{MATERIALS AND METHODS}

\section{Synthesis of $\beta$-hematin}

Hematin (ferriprotoporphyrin IX hydroxide) (H3505), was purchased from Sigma Milan, Italy. $\beta$ hematin $(\mathrm{BH})$ was synthesized as described by Slater et al. [3]. In short, $60 \mu$ moles of hematin were dissolved in $8 \mathrm{ml}$ of $0.1 \mathrm{M} \mathrm{NaOH}$, under $\mathrm{N}_{2}$ then precipitated by the addition of $49 \mathrm{mmol}$ of acetic acid. 


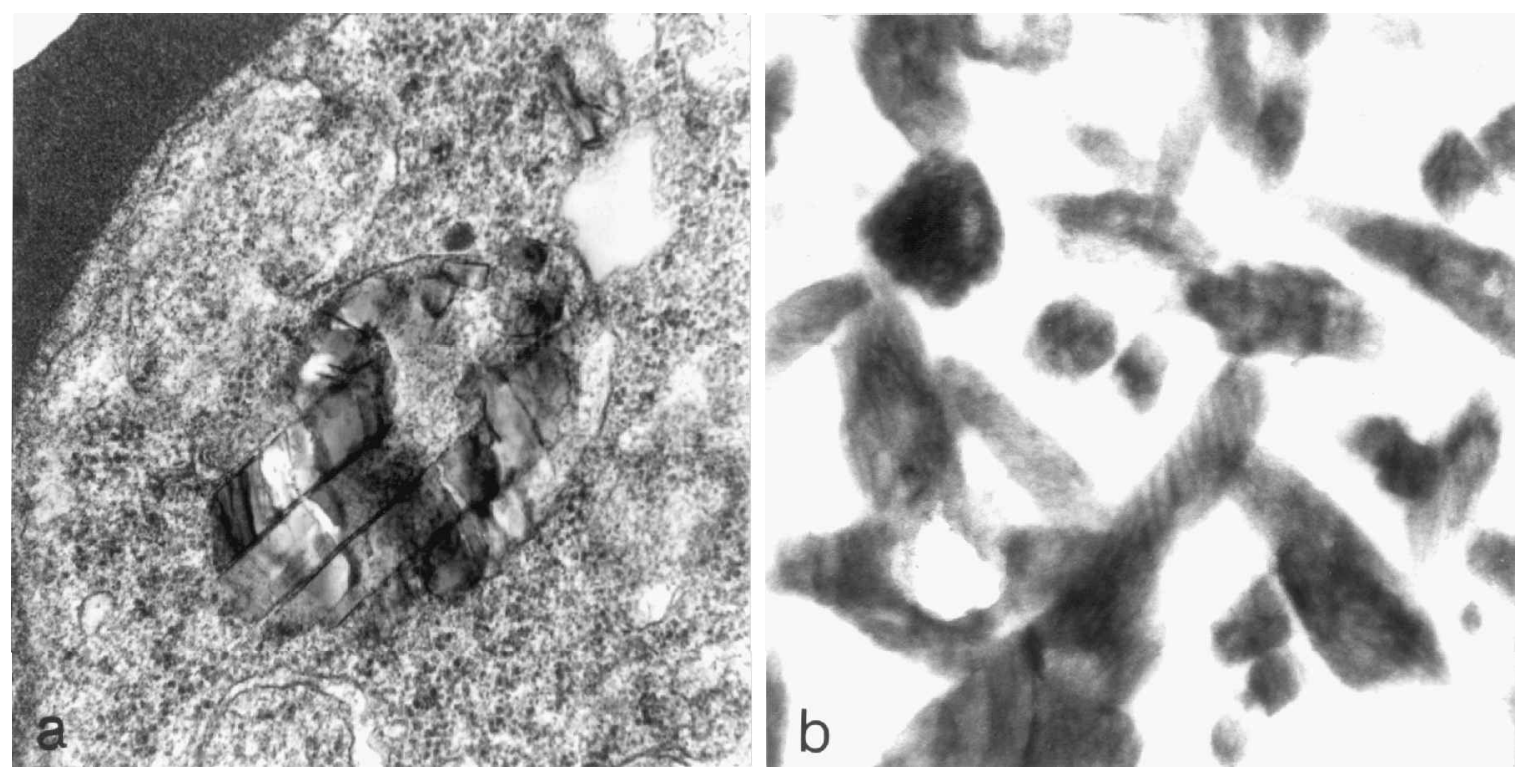

FIG. 1 Ultrastructure of native and synthetic hemozoin. Transversal banding was observed in both native hemozoin crystals found in the parasite FV (a) (magnification, $\times 71.000$ ) and in free particles of synthetic pigment ( $\beta$-hematin) (b) (magnification, $\times 71.000)$.

The suspension was heated overnight at $70^{\circ} \mathrm{C}$, and the precipitate was then centrifuged and washed four times with distilled water. Unreacted hematin was removed by extracting the precipitate twice for $3 \mathrm{~h}$ in $0.1 \mathrm{M}$ sodium bicarbonate buffer at $\mathrm{pH}$ 9.1. The remaining insoluble material was recovered by centrifugation, washed four times in distilled water and lyophilized. The characteristics of the final product were routinely controlled by infrared spectroscopy as reported elsewhere $[7,18]$. Batches of $\mathrm{BH}$ that did not meet standard criteria for purity were discarded. To facilitate the treatment of cell monolayers with insoluble $\mathrm{BH}$, the compound was resuspended in the culture medium, mechanically microdispersed, and then sonicated.

\section{Parasite cultures}

Laboratory-adapted $P$. falciparum parasites (NF54 strain) were cultured in vitro in RPMI 1640 in human red blood cells (RBCs) according to Druilhe [19].

\section{Purification of native hemozoin}

Native hemozoin from Plasmodium falciparum in vitro cultures was a kind gift from Prof. S. Picot, University of Lyon, France. Parasitized red blood cells containing more than $10 \%$ schizont forms were collected and washed twice with PBS. They were lysed with $200 \mu \mathrm{l}$ of $10 \%$ saponine in PBS for $15 \mathrm{~min}$ at $37^{\circ} \mathrm{C}$ and then washed three times in cold PBS. Crude hemozoin was then extracted by incubation for $1 \mathrm{~h}$ at $55^{\circ} \mathrm{C}$ using a lysing buffer $(50 \mathrm{mM}$ tris $\mathrm{HCl}$, $\mathrm{pH} 8,10 \mathrm{mM}$ EDTA, $1 \%$ SDS, $10 \mathrm{mg} / \mathrm{ml}$ proteinase K). After centrifugation, the preparation was washed two times in PBS-EDTA and then exhaustively with distilled water.

\section{Cell cultures}

\section{Monocytes}

Human monocytes (MN) from healthy blood donors were separated from total blood by centrifugation on Ficoll-hypaque solution (Pharmacia, Sweden). MNs were further separated from Iymphocytes by adherence to fibronectin-coated dishes (24well culture plates) for $3 \mathrm{~h}$ at $37^{\circ} \mathrm{C}$ and then washed to remove non-adherent cells. Approximately $5 \times 10^{5}$ adherent cells per well were obtained with an $\mathrm{MN}: \mathrm{RBC}$ ratio of $1: 40$.

J774 cells

A murine macrophage-like cell line (J774) [20] was maintained in MEM (Gibco-BRL-Life technologies) supplemented with $10 \%$ heatinactivated FBS (HyClone), 1\% glutammine, 1\% non-essential amino acid, $2 \%$ tricine and $1 \%$ penicillin-streptomycin in $5 \% \mathrm{CO}_{2}$ at $37^{\circ} \mathrm{C}$. $\mathrm{J} 774$ macrophages were mechanically collected with a cell lifter (Costar Italia, Milan, Italy) and transferred to a fresh medium every 3-4 days.

\section{Electron microscopy}

Specimens were fixed at 24 and $48 \mathrm{~h}$ of culture for MNs and at different times (25 $\mathrm{min}$ and $4 \mathrm{~h}$ ) for J774 macrophages after treatment with $\beta$-hematin, native hemozoin, or carbonyl iron particles. Longer incubation times were not suitable for $\mathrm{J} 774$ cells due 

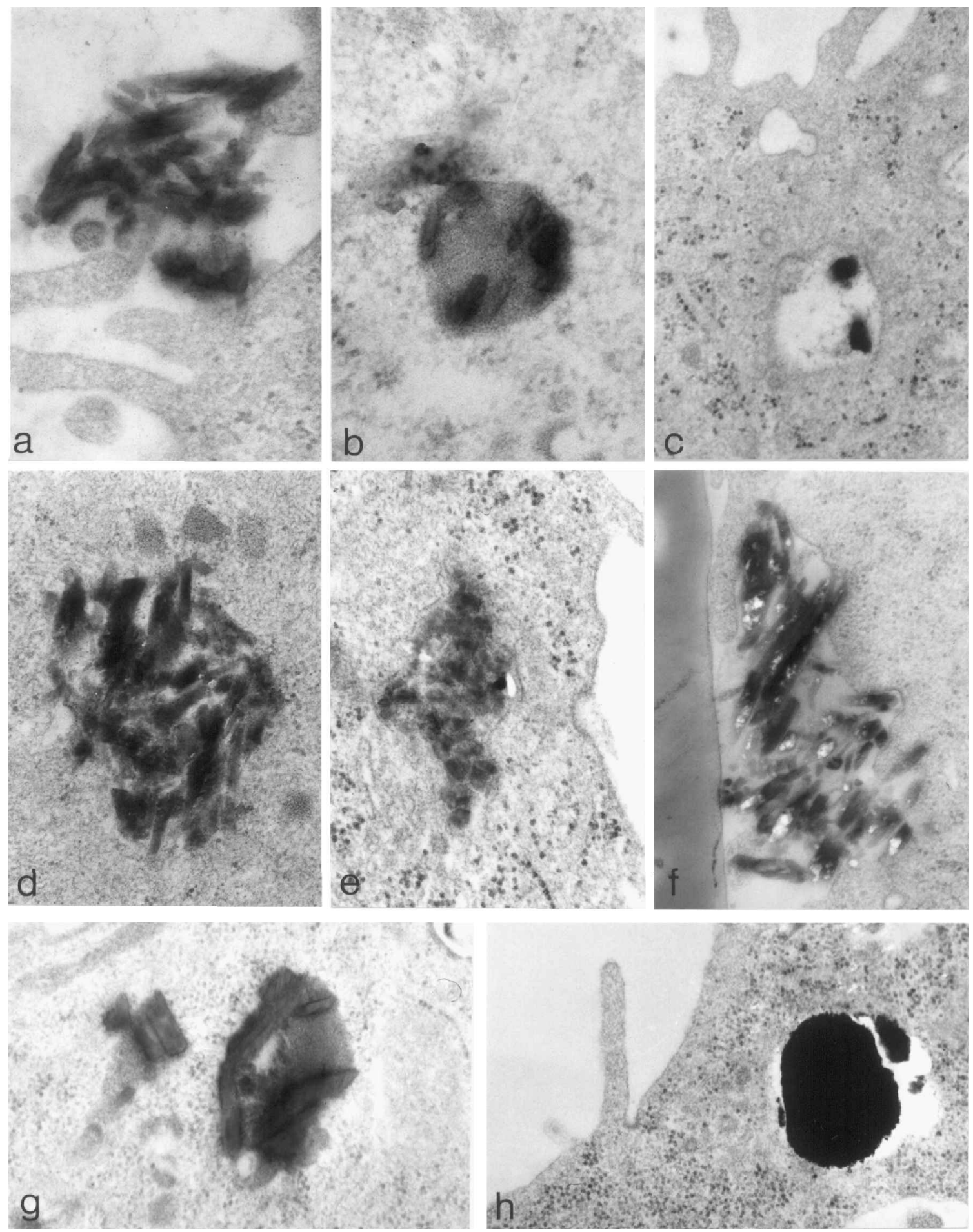

FIG. 2 Electron micrographs of the uptake of synthetic and native hemozoin particles and carbonyl iron fillings by $J 774$ macrophages. (a) An extracellular cluster of $\beta$-hematin crystals adhering to the plasma membrane and to microvilli of a macrophage.

Magnification, $\times 40.000$. (b) Several $\beta$-hematin particles in dense phagolysosome. Magnification, $\times 68.000$. (c) Two small $\beta$-hematin particles in an early phagosome. Magnification, $\times 40.000$. (d) A cluster of free, non-membrane bound intracytoplasmic $\beta$-hematin particles. Magnification, $\times 45.000$. (e) A cluster of $\beta$-hematin particles surrounded by a discontinuous membrane. Magnification, $\times 43.000$. (f) An extracellular cluster of $\beta$-hematin particles which appear to perforate the plasma membrane of a macrophage. Magnification, $\times 40.000$. (g) Native hemozoin crystals either freely scattered in the cytoplasm or within a dense phagolysosome.

Magnification, $\times 36.000$. (h) Intracytoplasmic carbonyl iron particles limited by a cloar halo. Magnification, $\times 29.000$. 

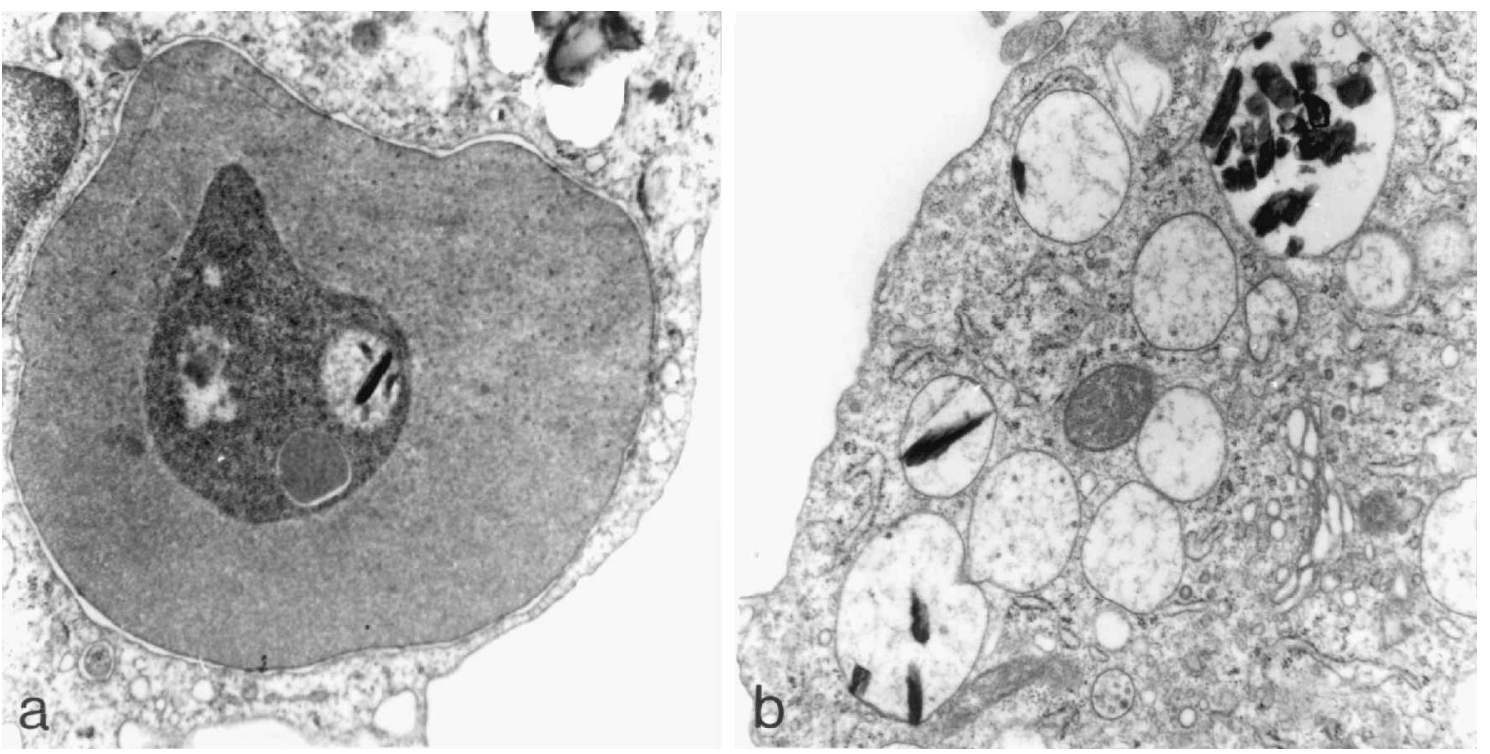

FIG. 3 Phagocytosis of P. falciparum-infected red blood cells. (a) Human monocyte with well-developed Golgi cisterane and numerous lysosomes and an infected RBC with hemozoin crystals in the FV. Magnification, $\times 20,000$. (b) A human monocyte containing lysosomes and phagolysosomes with remnants of RBC lysis and abundant hemozoin crystals. Magnification,$\times 30.000$.

to their fast growth rate in vitro. Cells were fixed with $2.5 \%$ glutaraldehyde in $0.1 \mathrm{M}$ phosphate buffer $(\mathrm{pH} 7.2)$ for $1 \mathrm{~h}$ at $25^{\circ} \mathrm{C}$, post-fixed with osmium tetraoxide, dehydrated in ethanol and embedded in situ with Epon 812. Sections were cut perpendicularly to the substrate and stained with uranyl acetate and lead citrate.

\section{RESULTS}

In the parasite $\mathrm{FV}$, hemozoin appears as polygonal crystals that, as the parasite matures, tend to stack upon each other, giving rise to larger structures with apparent transverse banding (Figure 1a). Such findings are comparable to those observed with synthetic pigment (Figure 1b).

When J774 macrophages were cultured in the presence of synthetic hemozoin, aggregates of crystal particles were seen to adhere to the cell plasma membrane (Figure 2a) and were then taken up, apparently via an endocytotic lysosomal process. Within 25 min of treatment, aggregates of small crystals were observed, mostly inside electron-dense phagolysosomes (Figure $2 \mathrm{~b}$ ), and more rarely, in early lysosomes with a clear matrix (Figure 2c).

Larger clusters of crystal particles were also observed free in the cytoplasm with no apparent delimiting membrane (Figure $2 \mathrm{~d}$ ) or surrounded by a discontinuous membrane (Figure 2e). Occasionally, synthetic hemozoin particles appeared to perforate the plasma membrane, thus penetrating into the macrophage via an endocytosis-independent pathway (Figure 2f). Similar patterns were observed with parasite-derived pigment (Figure $2 \mathrm{~g}$ ).

Macrophages fed with carbonyl iron (iron fillings) showed intracellular particles surrounded by a clear halo but no visible delimiting membrane (Figure $2 \mathrm{~h}$ ).

Monocytes co-cultured with $P$. falciparuminfected RBCs showed signs of activation, including an increased number of lysosomes, phagolysosomes, and more developed Golgi cisternae (Figures 3a and $3 b)$ as compared to control monocytes. The various steps of the phagocytosis of infected RBCs were observed, with no apparent preference for a specific parasite stage. The only intra-monocyte structure that could be positively ascribed to the parasite after $24 \mathrm{~h}$ of culture was the pigment. Crystals were found inside vacuoles with a single delimiting membrane. Morphologically, crystals appeared to be identical to those found in intra-erythrocytic parasites (Figures $3 a$ and $3 b)$.

\section{DISCUSSION}

Synthetic $\beta$-hematin is morphologically indistinguishable from native hemozoin, whether it resides inside the parasite FV or is extracted from parasite cultures. The pictures are similar to those obtained using different materials and techniques $[1,6,21$, 22].

The uptake of free particles partially follows the classical receptor-dependent endocytotic pathways, by which substances adhere to specific plasma membrane receptors and then are internalized through pinching-off of clathrin-coated vesicles, and incorp- 
orated into phagolysosomes. In our samples, obvious coated pits internalizing pigment particles are never observed. Instead, small crystal aggregates are observed in rare early phagosomes and numerous phagolysosomes. A more frequent finding is that of larger clusters of crystal particles scattered in the cytoplasm, without or with an incomplete delimiting membrane. This bears similarity to the uptake of inert material such as asbestos fibers. It has been proposed that, when ingested by lung alveolar macrophages, fibers would at first be free in the cytoplasm, and subsequently surrounded by a delimiting membrane and degraded by a phagolysosome.

The mechanism of transport of inert materials through the plasma membrane leading to the formation of clusters of free intracytoplasmic particles is still largely unknown. Our findings indicate that hemozoin could enter cells through plasma membrane ulcerations, as is the case for asbestos [2325].

By contrast to free crystal particles, the ingestion of whole parasites results in single membrane-bound pigment. Morphologically, such structures are indistinguishable from the parasite FV.

The ingestion of hemozoin is known to affect and modulate the phagocyte response to inflammatory stimuli, although conflicting results are reported as to the direction of change with different cell types and pigment preparations [5, 15, 16, 26, 27]. Biochemical studies ascribe such variability to the cells' different membrane lipid composition and antioxidant defenses [20]. The findings of this study indicate that native and synthetic pigment are internalized by phagocytes in the same way. They also indicate that there may be differences between the ingestion of free particles and whole parasites. Studies are underway to characterize the modulation of phagocytes' functions in this latter case.

\section{REFERENCES}

1. Ashong J, Blench I, Warhurst D. The composition of haemozoin from Plasmodium falciparum. Trans Royal Soc Trop Med Hyg. 1989;83:167-172.

2. Fitch $C D$, Kanjananggulpan $P$. The state of ferriprotoporphyrin IX in malaria pigment. J Biol Chem. 1987;262:15552-15555.

3. Slater AFG, Swiggard WJ, Orton BR, Flitter WD, Goldberg $\mathrm{DE}$, Cerami A. An iron-carboxilate bond links the heme units of malaria pigment. Proc Natl Acad Sci USA. $1991 ; 88: 325-329$.

4. Goldie P, Roth E, Oppenheim J, Vandergerg J. Biochemical characterization of Plasmodium falciparum hemozoin. Am J Trop Med Hyg. 1990;43:584-596.

5. Taramelli D, Basilico N, Pagani E, Grande R, Honti D, Ghione $M$, Olliaro $P$. The heme moiety of malaria pigment $(\beta-$ hematin) mediates the inhibition of nitric oxide and tumor necrosis factor- $\alpha$ production by lipopolysaccharide-stimulated macrophages. Exp Parasitol. 1995;81:501-511.

6. Adams P, Egan T, Ross D, Silver J, Marsh P. The chemical mechanism of $\beta$-hematin formation studied by Mössbauer spectrocopy. Biochem J. 1996;318:25-27.

7. Basilico N, Monti D, Olliaro P, Taramelli D. Non-iron porphyrins inhibit beta-hematin (malaria pigment) polymerization. FEBS Letters. $1997 ; 409: 297-299$.
8. Duarte MI, Corbett CE, Boulos M, Amato N. Ultrastructure of the lung in falciparum malaria. Am J Trop Med Hyg. $1985 ; 34: 31-35$.

9. Pongponratn E, Riganti M, Harinasuta T, Bunnag D. Electron microscopic study of phagocytosis in human spleen in falciparum malaria. Southeast Asian J Trop Med Public Health. $1989 ; 20: 31-39$.

10. Wickramasinghe $S$, Phillips $R$, Looareesuwan $S$, Warree DA, Hughes $M$. The bone marrow in human cerebral malaria: Parasite sequestration within sinusoids. $\mathrm{Br} \mathrm{J}$ Haematol. $1987 ; 66: 295-306$.

11. Amodu O, Adeyemo A, Olumese P, Gbadegesin R. Intraleucocytic malaria pigment and clinical severity of malaria in children. Trans Royal Soc Trop Med Hyg. 1998;92:54-56.

12. Phu NH, Day NP, Diep PT, Ferguson DJP, White NJ. Intraleucocytic malaria pigment and prognosis in severe malaria. Trans Royal Soc Trop Med Hyg. 1995;89:200-204.

13. Metzger WG, Mordmuller BG, Kremsner PG. Malaria pigment in leukocytes. Trans Royal Soc Trop Med Hyg. $1995 ; 89: 637-638$.

14. Sullivan AD, Meshnick SR. Haemozoin identification and quantification. Parasitology Today. $1996 ; 112: 285-294$.

15. Arese $P$, Schwarzer E. Malarial pigment (haemozoin) : a very active "inert" substance. Ann Trop Med Parasitol. $1997 ; 91: 501-516$.

16. Pichyangkul S, Saengkrai P, Webster H. Plasmodium falciparum pigment induces monocytes to release high levels of tumor necorsis factor- $\alpha$ and interleukin-1 $\beta$. Am J Trop Med Hyg. $1994 ; 51: 430-435$.

17. Taramelli D, Basilico N, De Palma A, Saresella M, Ferraute P, Musson. The effect of synthetic malaria pigment (betahematin) on adhesion molecule expression and interleukin-6 production by human endothelial cells. Trans Royal Soc Trop Med Hyg. 1998;92:57-62.

18. Egan T, Ross D, Adams P. Quinoline anti-malarial drugs inhibit spontaneous formation of $\beta$-haematin (malaria pigment). FEBS Letters. 1994;352:54-57.

19. Druilhe $P$, Jacquier $P$, Lambert $D$, Gentilini M. P. falciparum in vitro cultures: Improvement using umbilical cord serum and medium modification. Tropenmedizin und Parasitologie. 1980;31:825-864.

20. Omodeo-Sale F, Basilico N, Folini M, Olliaro P, Taramelli D. Macrophage populations of different origins have distinct susceptibilities to lipid peroxidation induced by $\beta$-haematin (malaria pigment). FEBS Letters. 1998;433:215-218.

21. Olliaro P, Castelli F, Milano F, Filice G, Carosi G. Ultrastructure of Plasmodium falciparum "in vitro". I. Base-line for drug effects evaluation. Microbiologica. $1989 ; 12: 7-14$

22. Goldberg DE, Slater AFG, Cerami AH. Hemoglobin degradation in the malaria parasite Plasmodium falciparum: An ordered process in a unique organelle. Proc Natl Acad Sci USA. 1990;87:2931-2935.

23. Fasske E. Pathogenesis of pulmonary fibrosis induced by chrysolite asbestos: Longitudinal light and electron microscopic studies on the rat model. Virchows Arch (Pathol Anat). 1986;408:329-346.

24. Davis J. The effect of chrysolite asbestos dust on lung macrophages maintained on organ culture: An electron-microscope study. Br J Exp Pathol. $1967 ; 48: 379-385$.

25. Churg $A$. The uptake of mineral particles by pulmonary epithelial cells. Am J Respir Crit Care Med. $1996 ; 154: 1124-1140$.

26. Sherry B, Alava G, Tracey K, Martiney J, Cerami A, Slater A. Malaria-specific metabolite hemozoin mediates the release of several potent endogenous pyrogens (TNF, MIP1 $\alpha$, and MIP-1 $\beta$ ) in vitro and altered thermoregulation in vivo. $J$ Inflamm. 1995;45:85-96.

27. Prada J, Malinowsky J, Muller S, Bienzle U, Kremsner P. Hemozoin differentially modulates the production of interleukin 6 and tumor necrosis factor in murine malaria. Eur Cytokine Netw. $1995 ; 6: 109-112$. 